\title{
THE WET COVERAGE - HOW WELL DO WE DO?
}

\author{
Jan-Erik Solheim ${ }^{1,2}$, \\ 1 Universitetet $i$ Tromsø, Insitutt for Fysikk, Nordlysobservatoriet, $N$ - \\ 9037 Tromsø, Norway \\ 2 present: Wilh Wilhelmsen vei 71, N-1263 Hosle, Norway
}

Received December 5, 2002

\begin{abstract}
The Whole Earth Telescope collaboration is build solidly on the interest of the participants. One of the goals of the collaboration is to produce a high signal to noise, as continuous as possible, light curve for a selected target. During the nearly 15 years of existence the operation of the network has been based on what the members have been able to provide of local funds for their own participation, in addition to NSF grants to run the headquarters activities. This has led to a very uneven geographical distribution of participating groups and observatories. An analysis of the coverage of some of the last WET runs shows that we still have large holes in the coverage, and this leads to aliasing and loss of precision in our final products.
\end{abstract}

Key words: techniques: photometric-methods: observational, WET collaboration observations, network, global distribution

\section{INTRODUCTION}

\subsection{The beginning}

The Whole Earth Telescope, or WET, is an existing telescope that comes alive one or two times every year. It has done so since XCov01 in 1988, and at the time of this workshop, we have just finished our XCov22. How it all started, based on the ideas of Ed Nather, about "temporal spectroscopy" is well described in the proceedings of the first WET workshop, which was held in honour of Ed Nather's 65th anniversary in Austin, Texas in November 1991 (Solheim 1993), and in his own recollection of the first years of WET at the second workshop at the Moletai Observatory (Nather 1993). At that time 9 XCovs had been performed, and a healthy influx of 
non-Texan astronomers in the project had started. Today the original Texans are a minority in the collaboration, but the basic idea of how we do our campaigns is the same.

The first XCov took place in 1988 and produced nearly 240 hours of white-light photometry of PG $1346+082$ (CR Boo). The analysis of this highly variable interacting binary system proved to be quite difficult and was finally published in 1997 (Provencal et al. 1997). The author list of this publication has 18 names of which 12 are "Texans" in the meaning staff or graduate students of the Department of Astronomy at University of Texas in Austin. Only two non-Texan co-authors from XCov01 are still active in the WET today: G. Vauclair and D. O'Donoghue. In XCov01 only 6 observatories participated: MacDonald in Texas, Siding Spring in Australia, Cerro Tololo in Chile, Mauna Kea in Hawaii, SAAO in South Africa and Haute Provence in France. The window function of this entire WET run is given in Fig. 1. We see noticeable sidebands due to lack of coverage at certain longitudes. All the pioneer observatories used one or two channel photomultiplier photometers.

In the recently finished XCov22, 21 observatories participated, not all of them as yet "certified" WET members. 10 of the participants used CCDs for their photometry. Figure 2 shows a map

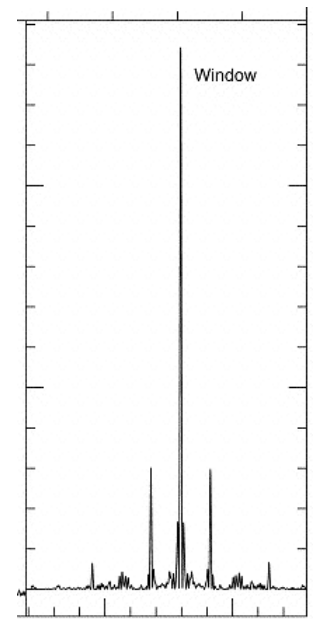

Fig. 1. The Fourier Transform Window for the entire WET light curve of CR Boo in XCov01 (from Fig. 2 of Provencal et al. 1997). The x-ticks are $10 \mu \mathrm{Hz}$ and the $y$-axis shows power. of the world indicating the first six WET observatories, and the others that have later participated one or more times. In total 24 observatories are shown in this map, in addition to the Hubble Space Telescope, which participated in XCovs 16, 17, 19 and 22. In some observatories, like McDonald, Hawaii, Itajuba, BAO, South Africa and La Palma, more than one telescope has been used for the WET. On the Canary Islands also the Teide Observatory has been frequently used.

\subsection{The basic ideas and weakness of the WET}

One of the cornerstones of the WET is that this is an organization of individual scientists, providing their own resources, who participate to the extent that they find the target challenging enough to spend their time and resources. 


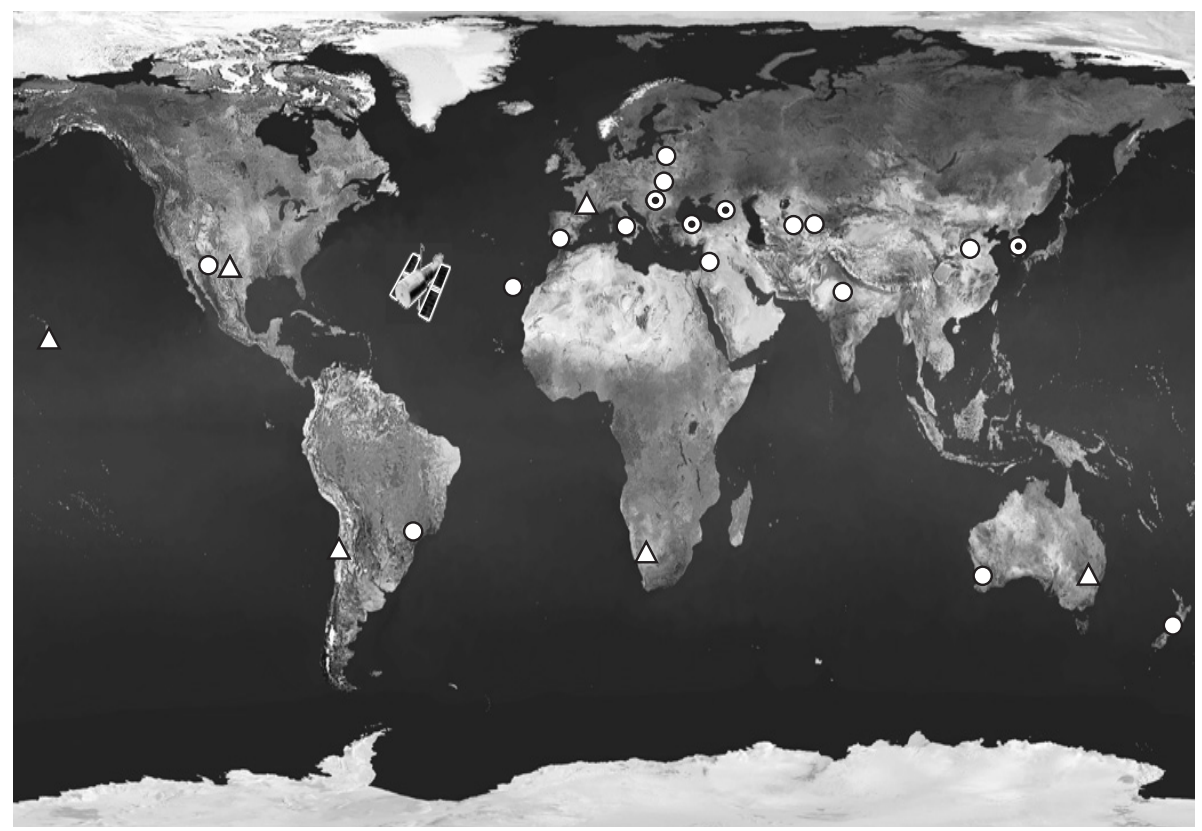

Fig. 2. The distribution of participating WET observatories around the world. The triangles are the six pioneer observatories, the open circles are observatories that have later participated, and the open circles with dots are the newcomers, not yet fully certified as WET observatories. The Hubble Space Telescope is also shown as it participated in XCovs 16, 17, 19 and 22 .

In the pioneer years even the organizing of campaigns and testing of instruments were left to individuals - and this led to some confusion and lack of communication. There are rumours that even some application deadlines were lost. Now this is corrected, by having one officer, a co-director in Iowa: Reed Riddle that takes care of the administrative work, and see to that deadlines are not forgotten and that we have the information needed to submit applications and do the observations correctly when the time comes. But the Director, the Committee that determines the target, and all the participants are scientists that also have other duties. So this is a science run co-operation - not an organization or a governmental funded observatory.

The goals of the WET are nicely presented in the paper by Nather et al. (1990) where the need for continuous coverage was clearly demonstrated. If we have a continuous light curve, we get a window function like a delta function, and even near small peaks in frequency space can be detected. We can, to some extent, repair holes in the light curve by the prewhitening processes and in 
some cases find peaks that may seem hidden in the noise, but if we have systematic holes in the light curve at certain longitudes, there becomes a limit to what we can deduce from the light curve.

One of the difficult places to get good coverage is over Asia. So far we have collaborators in India and China which participate on a regular basis. But due to the weather pattern they cannot provide data at certain periods of the year. To plug the gap a co-operation was started with E.G. Meištas and other colleagues in Vilnius who at that time had a telescope at Mt. Maidanak in Uzbekistan, and had good relations with other observatories in Central Asia which could be used (Meištas 1993). Unfortunately the easy access to Mt. Maidanak was lost during the process of nationalization in Uzbekistan, and attempts to use observatories in Kazakhstan (Mironov 1998) has failed due to the lack of money. In the next section I will present the results of an analysis of the coverage of some of the latest XCovs.

\section{THE COVERAGE IN SOME RECENT XCOVS}

A quick look at the quality of a WET campaign is to study the window function as it is made at the headquarters and posted on the internet as soon as the data are received. This may not be the final one, since some data do not reach the HQ while it functions, and the WET pages from a campaign are not updated after a campaign is finished. Also some data may be of such poor quality that it will not be included in the final analysis. Such an example is given in the paper of Kurtz et al. (2003 these proceedings).

So with the precaution that the final coverage may look different, I will in the following present the coverage of the three last full XCovs: no 19, 21 and 22 for the main targets. To illustrate the coverage I show in Table 1 how many observatories that participated in the various campaigns. Not all of them observed the main target. It is the job of the HQ to assign other observatories to secondary or tertiary targets when the first one is covered. From this table it is obvious that we have poor coverage over the Oceanic and Asian section. However, a good site as Hawaii counts as many of the poor European sites.

\subsection{Coverage in XCov19}

Figure 3 shows the coverage as we are used to see it in the internet presentation of the campaigns (http://wet.iitap.iastate.edu) The light curve panels for the main target GD 358, are from the first and second week, respectively. The left panel has long gaps between 
Table 1. Number of participating telescopes in recent XCov's distributed in longitude.

\begin{tabular}{|c|c|c|c|c|}
\hline $\mathrm{XCov}$ and date & $\begin{array}{c}60 \mathrm{E}-30 \mathrm{~W} \\
\text { Afr., Eur }\end{array}$ & $\begin{array}{l}30 \mathrm{~W}-120 \mathrm{~W} \\
\text { N.\& S. Am. }\end{array}$ & $\begin{array}{c}120 \mathrm{~W}-150 \mathrm{E} \\
\text { Hawaii, NZ }\end{array}$ & $\begin{array}{l}150 \mathrm{E}-60 \mathrm{E} \\
\text { Asia, Aust. }\end{array}$ \\
\hline 19: May/June 2000 & 7 & 3 & 2 & 1 \\
\hline 21: April 2001 & 9 & 6 & 1 & 3 \\
\hline 22: May 2002 & 11 & 5 & 1 & 4 \\
\hline
\end{tabular}

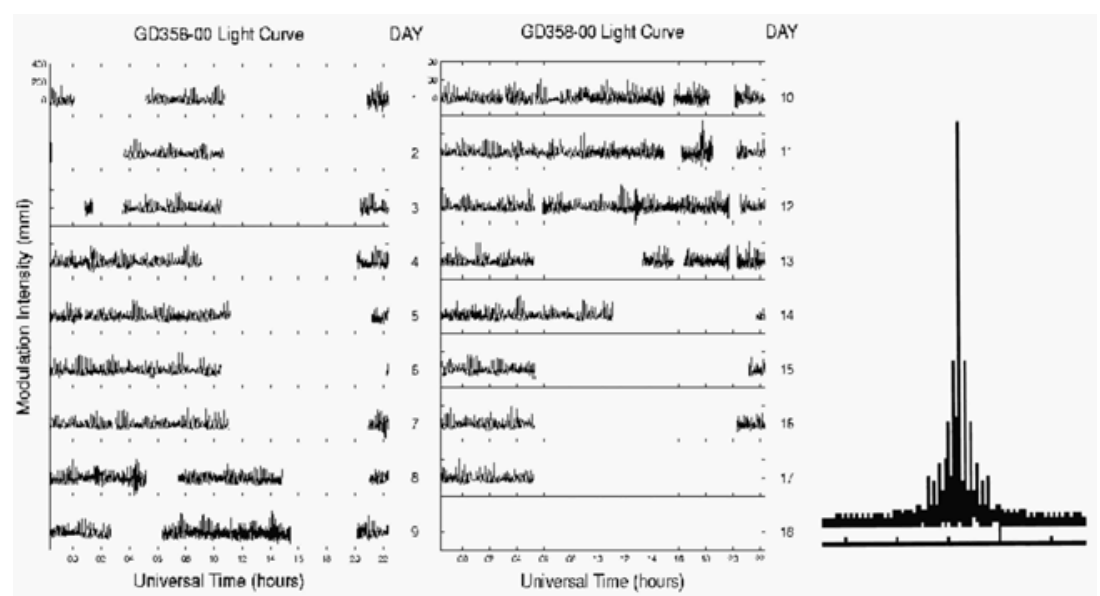

Fig. 3. This is the usual way for presenting in the internet the results from an ongoing WET campaign. In this example, XCov19 in May/June 2000 is shown. The left and center panels show the light curve from the first and second week of the campaign, and the rightmost panel is the resulting window function, in milliamplitude units.

10 and 20 UT indicating poor Asian coverage. In the second week there are three nights with almost complete coverage, days 10, 11 and 12, probably one of the best coverages in the history of the WET (central panel). The final window function is shown in the right hand panel, and we see that there is a forest of aliasing peaks in this function. It remains to be seen what the final analysis say about this data set (Kepler 2003, these proceedings).

\subsection{Coverage in XCov21}

This campaign took place in April, and was blessed with bad weather over most Europe and missing observatories in Asia. Still the redundancy over Europe/South Africa, and the sufficient coverage over the American continents made an excellent coverage of 15 nights or more between 20 UT and 09 UT as shown in Figure 4, for the main 


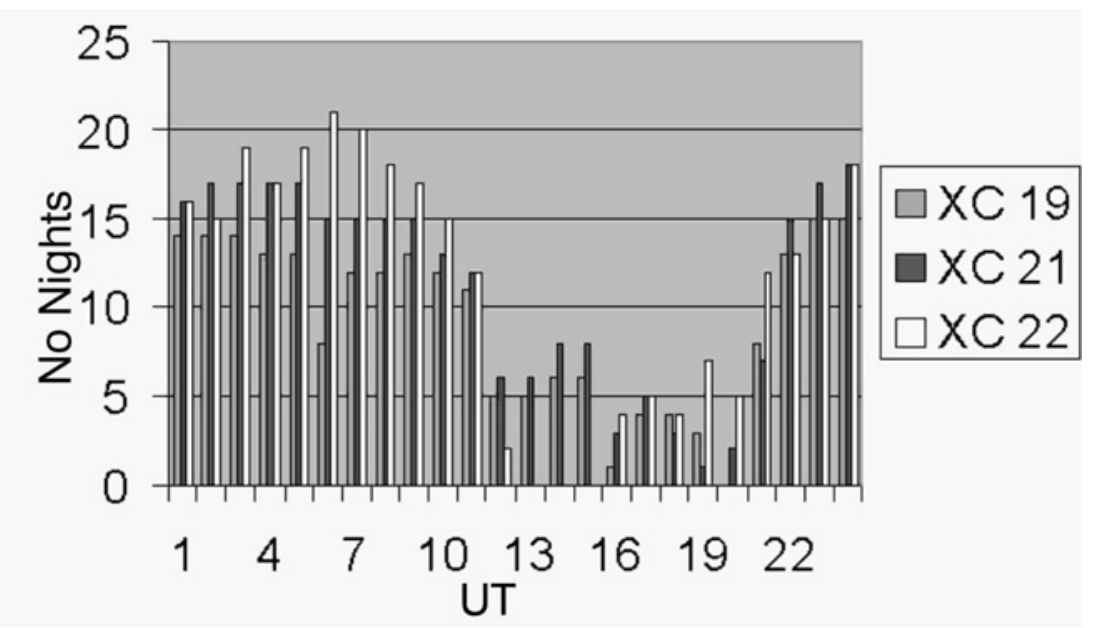

Fig. 4. The number of covered nights for the main targets in XCovs 19, 21 and 22 taken from the light curves presented in the WET campaign internet pages.

target PG 1336 .

\subsection{Coverage in XCov22}

This XCov collected an amazing number of 21 telescopes (Table 1) and this resulted in more than 15 nights coverage between 20 and 9 UT for the prime target PG $1456+103$, but almost no coverage between 10 and $13 \mathrm{UT}$, and poor coverage between 13 and $18 \mathrm{UT}$, as shown in Figure 4. The data presented on the web may in this case not be complete, since data from the Terskhol Observatory did not reach the $\mathrm{HQ}$ in time to be included in the on-line reduction which is also presented by Handler (2003, these proceedings).

\section{CONCLUSIONS}

The WET collaboration is very successful in acquiring data between $21 \mathrm{UT}$ and $11 \mathrm{UT}$ which corresponds to observatories in S. Africa, Europe and the Americas. In order to reach our goal of a complete light curve, we need better coverage over the Asian and Australian continents. This is a challenge for future campaign organizers.

ACKNOWLEDGMENTS. Special thanks are to E.G. Meištas for helping with the illustrations and his many efforts to help establishing observing stations in Central Asia. 


\section{REFERENCES}

Kepler S. O., Nather E.R., Winget D.E. et al. 2003, in The 6th WET

Workshop Proceedings (these Proceedings), eds. E. G. Meištas and J.-E. Solheim, Baltic Astronomy, 12, 45

Meištas E. G. 1993, Baltic Astronomy, 2,479

Mironov A. V., Tereshchenko V. M. 1998, Baltic Astronomy, 7, 351

Nather R. E., Winget D. E., Clemens J. C., Hansen C. J., Hine B. P. 1990, ApJ, 361, 309

Nather R. E. 1993, Baltic Astronomy, 2, 371

Provencal J. L., Winget D. E., Nather R. E. et al. 1997, ApJ, 480, 383

Solheim J.-E. 1993, Baltic Astronomy, 2, 363 